15

16

17

18

19

20

21

22

23

24

25

26

27

28

29

30

31

32

33

34

35

36

37

38

39

40

41

42

43

44

45

46

47

48

\title{
Deep learning-based tumor microenvironment segmentation is predictive of tumor mutations and patient survival in non-small-cell lung cancer
}

\author{
Łukasz Rączkowski ${ }^{1}$, Iwona Paśnik ${ }^{2}$, Michał Kukiełka ${ }^{1}$, Marcin Nicoś ${ }^{3}$, Magdalena A. Budzinska ${ }^{4}$, \\ Tomasz Kucharczyk $^{3}$, Justyna Szumiło ${ }^{2}$, Paweł Krawczyk ${ }^{3}$, Nicola Crosetto ${ }^{5,6}$, Ewa Szczurek ${ }^{1, *}$ \\ ${ }^{1}$ Faculty of Mathematics, Informatics and Mechanics, University of Warsaw, Poland \\ ${ }^{2}$ Department of Clinical Pathomorphology, Medical University of Lublin, Poland \\ ${ }^{3}$ Department of Pneumonology, Oncology and Allergology, Medical University of Lublin, Poland \\ ${ }^{4}$ Ardigen, Cracow, Poland \\ ${ }^{5}$ Division of Genome Biology, Department of Medical Biochemistry and Biophysics, Karolinska \\ Institutet, Stockholm, Sweden \\ ${ }^{6}$ Science for Life Laboratory, Stockholm, Sweden
}

*Correspondence should be sent to: ES (szczurek@ mimuw.edu.pl) 


\section{Abstract}

50 Despite the fact that tumor microenvironment (TME) and gene mutations are the main determinants of

51 progression of the deadliest cancer in the world - lung cancer - their interrelations are not well

52 understood. Digital pathology data provide a unique insight into the spatial composition of the TME.

53 Various spatial metrics and machine learning approaches were proposed for prediction of either patient

54 survival or gene mutations from these data. Still, these approaches are limited in the scope of analyzed

55 features and in their explainability and as such fail to transfer to clinical practice. Here, we generated

5623,199 image patches from 55 hematoxylin-and-eosin (H\&E)-stained lung cancer tissue sections and

57 annotated them into 9 different tissue classes. Using this dataset, we trained a deep neural network ARA-

$58 \mathrm{CNN}$, achieving per-class AUC ranging from 0.72 to 0.99 . We applied the trained network to segment

59467 lung cancer H\&E images downloaded from The Cancer Genome Atlas (TCGA) database. We used

60 the segmented images to compute human interpretable features reflecting the heterogeneous composition

61 of the TME, and successfully utilized them to predict patient survival (c-index 0.723) and cancer gene

62 mutations (largest AUC 73.5\% for PDGFRB). Our approach can be generalized to different cancer types

63 to inform precision medicine strategies.

\section{Introduction}

65 In clinical practice it is common to diagnose cancer based on hematoxylin-eosin (H\&E) stained images

66 obtained through biopsy or surgery ${ }^{1}$. Such images are routinely stored for each patient, so there is an

67 abundance of patient-specific, disease progression-relevant data that until recently has not been utilised at

68 scale in cancer research. This has changed with the advent of digital pathology and the development of

69 machine learning approaches to various predictive tasks based on H\&E image data $\stackrel{2,3}{\text {. }}$

71 In addition to tumor cells, $H \& E$ images portray the spatial architecture of the tumor microenvironment

72 (TME), including stromal cells, immune cells, and hypoxic/necrotic tissue areas and their reciprocal 
73 spatial arrangement. The TME plays an important role in cancer progression and metastasis, and thus it is

74 critical to study its composition extensively ${ }^{4}$. Different tumors, even of the same type, have various

75 genetic profiles resulting from gene mutations ${ }^{\underline{5}}$. For a given cancer type, survival of individual patients

76 can largely vary $\frac{6,7}{}$. Finally, the TME of different tumors is also different ${ }^{\frac{8}{*}}$. A burning question in this

77 context is how the structure of the TME relates to patient survival and gene mutations. This question is

78 particularly relevant for lung cancer. Lung cancer is the deadliest cancer type worldwide, and lung

79 adenocarcinoma (LUAD) is becoming the mainly diagnosed subtype of lung cancer ${ }^{9}$. LUAD includes a

80 relatively higher proportion of cases without tobacco exposure. Thus, it has a more balanced molecular

81 background and is more frequently associated with the presence of single somatic driver mutations that

82 may be effectively managed with specific molecularly targeted therapies ${ }^{\underline{10}}$. Several genes are known

83 markers of response to treatment and survival in LUAD, including EGFR, ALK, ROSI, BRAF, NTRKI-3,

$84 R E T, M E T, K R A S$, and diagnostic panels for targeted gene sequencing for detecting mutations in critical

85 genes are routinely used in clinical practice ${ }^{11}$. H\&E images are commonly inspected for LUAD diagnosis

86 and prognosis $\frac{12}{2}$. Wide tumor spread, access to vessels, large areas of necrosis visible in H\&E images, are

87 associated with poor diagnosis ${ }^{4}$, while abundance of immune cells indicates anti-tumor response of the

88 immune system and associates with better survival ${ }^{13,14}$. The TME plays an important role in LUAD

89 response to immunotherapy. Expression of PD-1 and PD-L1 on cancer or immune cells, as well as tumor

90 mutation burden (TMB), are important biomarkers of immune checkpoint inhibitor efficiency $\frac{15.16}{}$. The

91 interconnections between the spatial TME composition, gene mutations and LUAD patient survival are so

92 far not well understood.

93

94 Computational prediction of patient survival from H\&E images has been either performed based on

95 spatial metrics or using deep learning approaches. In the former case, spatial metrics are initially used to

96 summarize the spatial arrangement of different tissues and next their correlation with survival is

97 investigated. Alternatively, these spatial metrics are used as features in traditional machine learning

98 algorithms $\frac{17-20}{}$. The TME can be very heterogeneous, so it is not obvious how to quantify it and what 
99 metrics to use. These metrics include proportion-based ${ }^{21}$, clustering-based ${ }^{22}$, and methods borrowed from

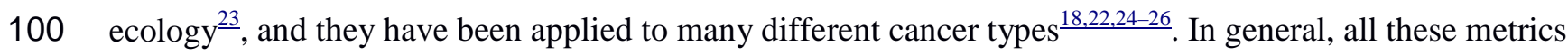

101 share a common trait, i.e., they incorporate only a limited number of tissue types at once, such as tumor

102 cells and lymphocytes, tumor cells and stroma, etc. This approach cannot comprehensively capture the

103 complexity of the TME. Thus, there is an unmet need for an encompassing spatial metric that would

104 consider many possible TME components at once. In the latter case, deep neural networks are trained to

105 predict patient survival directly from H\&E images. Such deep learning-based methods are increasingly

106 used for survival prediction and have been shown to perform comparably to or even better than spatial

107 metric-based approaches ${ }^{27,28}$. However, one major disadvantage of deep learning methods is the lack of

108 explainability. Due to the complicated structure of these models and number of parameters, it is not easy

109 to surmise which parts of the TME are the most important for patient survival. This creates a need for

110 explainable H\&E image-based survival models.

111

112 Numerous methods for predicting gene mutations from H\&E images were introduced and applied to a

113 spectrum of cancers $\frac{29-34}{\text {, }}$, showing that such approaches can reveal links between the TME composition

114 and mutations of selected genes. However, similarly to deep learning-based methods for patient survival,

115 these models take raw image data as input and directly predict the presence of mutations. As such, it is

116 hard to assess what parts of the TME are most predictive of a given mutation. A recent study proved that

117 human-interpretable features extracted from images segmented with deep learning methods can be

118 successfully applied to predict phenotypic expression $\frac{35}{}$. This suggests that the same approach can be

119 implemented to predict patient survival and gene mutations, which has not yet been explored. To address

120 these shortcomings, here we develop a framework for predicting survival and gene mutations in LUAD

121 patients based on H\&E images and using human-interpretable features. First, we train a deep learning

122 classifier and apply it to segment the H\&E images from 467 tumor LUAD samples into nine tissue

123 classes. Next, we compute two human-interpretable spatial features that describe the composition of the

124 TME in segmented H\&E slides. Finally, we use these features in combination with clinical data to predict 
125

126

127

128

129

130

131

\section{Clinical samples}

133 We obtained the formalin-fixed paraffin embedded (FFPE) tissue samples from 55 primary tumors of

134 lung cancer (35 lung adenocarcinoma, 20 lung squamous cell carcinoma). The material was derived from

135 FFPE surgical materials as well as from diagnostic (biopsy) procedures performed at Medical University

136

137

138

139

140

141

\section{Extraction and annotation of the training dataset for ARA-CNN}

143 We extracted the training dataset from the H\&E slides sourced from 55 lung cancer patients. We loaded

144 these slides into the QuPath software package ${ }^{36}$ and regions of contiguous tissue were annotated by an

145 expert pathologist with the following nine labels: tumor, stroma, mixed, immune, vessel, bronchi,

146 necrosis, lung, background. The annotated regions were then traced by a moving window, which cut out

147 non-overlapping square patches of tissue with side size of $87 \mu \mathrm{m}$. In addition to $87 \mu \mathrm{m}$, we also tested the

148 training performance for patches with sizes of $74 \mu \mathrm{m}$ and $100 \mu \mathrm{m}$ (see Sup. Table 1-3). They resulted in 
149 worse performance (mean accuracy $84.64 \%$ for $74 \mu \mathrm{m}, 84.35 \%$ for $100 \mu \mathrm{m}, 85.21 \%$ for $87 \mu \mathrm{m}$ ), so we

150 proceeded with using the $87 \mathrm{um}$ sized ones. This gave us an initial version of the training dataset, which

151 was then improved upon by utilizing human-in-the-loop active learning, as part of the previously

152 proposed accurate, reliable and active (ARA) image classification framework ${ }^{37}$. In total, we ended up with

15323199 patches, divided in the following manner: 3311 tumor patches, as well as 1511 stroma, 716 mixed,

1541196 immune, 1236 vessel, 2030 bronchi, 4448 necrosis, 6031 lung, and 2211 background patches.

\section{ARA-CNN model}

157 The main component of the ARA framework is ARA-CNN, a Convolutional Neural Network (CNN)

158 architecture inspired in part by Microsoft ResNet ${ }^{38}$ and DarkNet $19^{\frac{39}{}}$. It includes standard techniques for

159 such models: Batch Normalisation ${ }^{40}$ (for normalisation and to reduce overfitting) and dropout ${ }^{41}$ (reduces

160 overfitting). The latter allowed us to apply variational dropout ${ }^{42}$ during testing. Variational dropout is

161 used to estimate uncertainty for every input image, which is returned together with its predicted class.

162

163 The model was trained in several iterations, each one improving upon the previous ones. After the first

164 training process, the distribution of uncertainty for images in each class was measured separately and due

165 to higher median uncertainty, it was concluded that there are three classes in need of more training

166 examples: mixed, vessel and bronchi. These were passed on to the pathologist, who labeled new regions

167 belonging to these classes. The resulting new training samples were extracted and added to the previous

168 training dataset. This adaptive training procedure was repeated three times, where each time the

169 uncertainty for each class was measured, until it was decided that the uncertainty results were at a

170 satisfactory level.

171

172 For training the final ARA-CNN model on the lung cancer tissue patches, we used stratified 10-fold

173 cross-validation. In each fold, the whole dataset of 23199 images was split into a training dataset and a

174 test dataset used for evaluation. The test dataset contained 2316 patches, while the training dataset 
175 consisted of 20883 patches. Each class was split in exactly the same proportion: $10 \%$ were sent to the test

176 dataset and $90 \%$ to the training dataset.

177 Additionally, in each training epoch the training data was split into two datasets: the actual training data

178 and a validation dataset. The latter was used for informing the learning rate reducer - we monitored the

179 accuracy on the validation set and if it stopped improving, the learning rate was reduced by a factor of

180 0.1. This split was in proportion $90 \%$ to $10 \%$ between actual training data and the validation set,

181 respectively.

182 For parameter optimisation, we used the Adam $^{43}$ optimiser. The training time was set to 100 epochs. The

183 training data was passed to the network in batches of 32 , while the validation and test data was split into

184 batches of 128 images. The loss function used during training was the categorical cross-entropy.

\section{TCGA image data segmentation using ARA-CNN}

186 The normalized patches (Supplementary Methods) served as input to ARA-CNN. For each input patch,

187 the model returned a classification probability into each of the nine predefined classes. With these results,

188 each patch was labeled with the class with the highest probability and then the labeled patches were

189 merged back into their full respective slides and colored by the label. This created segmented slides, with

190 clearly visible continuous areas of differing tissue.

191

192 The segmented slides were also validated by an expert pathologist, who assessed that 39 slides needed to

193 be excluded from further analysis. There were two reasons for that. The first one involved erroneous

194 classifications returned by ARA-CNN - 21 out of 506 slides contained errors of such nature. The other 18

195 slides were excluded due to markings and other staining errors. After this process, the final dataset

196 contained 467 slides.

197

198 


\section{Quantification of spatial features for the segmented tumor tissues}

200 The obtained segmented images from TCGA were then processed further in order to extract spatial

201 information in the form of two types of features: tissue prevalence (TIP) and tumor microenvironment

202 composition (TMEC). TIP is a distribution of tissue classes within the whole tissue area, i.e. excluding the

203 background class. TMEC measures a distribution of tissues that neighbour the tumor tissue within a

204 predefined margin.

205 The prevalence $t_{i}$ of tissue $i$ is expressed as:

206

$$
t_{i}=\frac{n_{i}}{N}
$$

207

208

209

210

211

212

213

214

215

216

217

218

219

220

221

222

223

224

225

where $n_{i}$ is the number of patches for tissue $i$ and $N$ is the total number of tissue patches (excluding the background class) and

$$
i \in\{T U M O R, \text { STROMA, MIXED, IMMUNE, VESSEL, BRONCHI, NECROSIS, LUNG }\} \text {. }
$$

The vector $T$ with entries given by $t_{i}$ makes up the TIP features. The background class was omitted, as it's not relevant to the tissue structure.

The microenvironment composition $m_{j}$ for tissue $j$ is:

$$
m_{j}=\frac{b_{j}}{B}
$$

where $b_{j}$ is the number of patches of class $j$ that neighbour the tumor class and $B$ is the total number of all patches neighbouring the tumor class (excluding the tumor itself and the background class), with

$$
j \in\{\text { STROMA, MIXED, IMMUNE, VESSEL, BRONCHI, NECROSIS, LUNG }\} \text {. }
$$

The TMEC features are organized in a vector $M$, with $m_{j}$ as its entries. The neighbour patches are considered only within a margin around the borders of tumor regions (see Figure 1b.). Each tumor patch is considered separately and up to eight neighbours around it are counted. These patches are summed up 
226

227

228

229

230

231

232

233

234

235

236

237

238

239

240

241

242

243

244

245

246

247 For each classification task, where the class was specified by the presence of mutation of a given gene,

248

249

250

to $b_{j}$ for each class $j$ and to $B$ in total.

Using the microenvironment and prevalence data, we also calculated three spatial metrics that were previously defined in the literature: intra-tumor lymphocyte ratio (ITLR) $)^{21}$, Simpson diversity index ${ }^{44}$, Shannon diversity index ${ }^{45}$. We used a simplified version of these metrics - instead of cell-wise, we calculated them patch-wise. Specifically, these metrics were computed as follows:

where $b_{I M M U N E}$ is the number of immune patches that neighbour the tumor and $n_{T U M O R}$ is the number of tumor patches in the whole slide.

\section{Mutation classification}

The processed data from TCGA served as input in the mutation classification task. The predictor variables consisted of clinical data (age, sex, smoking status, pathologic stage), the previously introduced ITLR, Shannon diversity index, Simpson diversity index, as well as the here proposed TMEC and TIP. The response variables were binary and were defined by the mutation status for the following genes: $A L K$, BRAF, DDR2, EGFR, KEAP1, KRAS, MET, PDGFRB, PIK3CA, RET, ROS1, STK11, TP53.

the dataset was oversampled so that positive (mutation occurred) and negative (mutation did not occur) subsets of examples were equal in size. Oversampling was done by inserting multiple copies of the positive examples so that their number reached that of the negative ones. 
252 Several combinations of predictors were tested: clinical, clinical + ITLR, clinical + Shannon diversity

253 index, clinical + Simpson diversity index, clinical + TMEC, clinical + TIP, clinical + TMEC + TIP,

254 TMEC only. To classify the mutation status for each gene, two distinct machine learning models were

255 trained and compared. The first one was a simple linear model in the form of logistic regression. It was

256 fitted using the Liblinear solver $\frac{46}{}$, with the L2 penalty and up to 2000 iterations. The second one was the

257 Random Forest algorithm ${ }^{47}$. We used the implementation from the sklearn Python library $y^{48}$ with default

258 parameter values.

259

260 All models were trained 100 times with 10-fold cross-validation and the resulting classification accuracy

261 metrics were averaged. Classification accuracy was evaluated using the AUC metric.

262

\section{Survival prediction data exploration}

264 For the survival prediction task, we defined the set of predictors as the same variables (clinical and

265 spatial) as in the mutation classification task, extended by the set of variables that indicated the mutations

in genes. The response variables consisted of the time to the last follow up and the censoring status for the patients. The latter was sourced from TCGA using the curated TCGA Data R package ${ }^{49}$.

268

269 We explored the univariate relations between the components of TIP and TMEC and survival, as well as

270 between the gene mutation status and survival, using the Kaplan-Meier estimator. For each tissue type $i$,

271 we compared survival between the groups with high and low $t_{i}$. Similarly, we compared survival between

272 the groups with high and low $m_{\mathrm{j}}$. The cutoff point in each case was chosen automatically by the survminer

273 R package. For gene mutations, we divided the patients based on the binary mutation status. The

274 significance of each survival comparison was assessed with the log-rank test.

275

\section{Survival modeling}

277 The aforementioned predictors were used as input to the Cox proportional hazards model. They were 
278 organized into the following basic variants: clinical, clinical + ITLR, clinical + Shannon diversity index,

279 clinical + Simpson diversity index, clinical + TMEC, clinical + TIP, clinical + TMEC + TIP. In addition,

280 variants with mutation data added on top of clinical data were considered. Each variant was trained in a

281 10-fold cross-validation schema and for each a resulting median c-index was measured. On top of that, we

282 also plotted hazard ratios for two models with the best c-index. For categorical variables, the hazard ratio

283 of their basal values is set to 1 . For the sex variable, the basal value was 'Female'. For the Stage variable,

284 the basal value was 'Early stage'. For mutation variables (EGFR, STK11 and TP53) the basal value was

285 the absence of alteration. Finally, for smoking status, non-smoker was set as basal.

286 Results

\section{Implementation and validation of ARA-CNN}

288 To automatically segment H\&E images and be able to compute human interpretable spatial features, we

289 first generated a training dataset consisting of whole slide scans of H\&E stained tissue sections from 55

290 LUAD samples from a single institution (Fig. 1a and Materials and methods). We annotated consistent

291 regions of tissue, marking them as one of the following nine classes: tumor with neoplastic epithelial

292 cells; stroma composed of connective tissue within tumor or extra-tumoral connective tissue; mixed

293 where connective tissue was strongly infiltrated with immune cells; immune composed of lymphocytes

294 and plasma cells or fragments of pulmonary lymph nodes; vessel composed of smooth muscle layers

295 (veins and arteries) with red blood cells within lumen; bronchi composed of cartilage and bronchial

296 mucosa; necrosis including necrotic tissue or necrotic debris; lung (lung parenchyma); and background of

297 the tissue scan (no tissue). We then extracted $87 \times 87 \mu \mathrm{m}$ tissue patches from each annotated region and

298 used them to iteratively train our previously introduced accurate, reliable and active convolutional neural

299 network (ARA-CNN) model, in each iteration obtaining additional annotations for classes pointed as

300 uncertain by the model ${ }^{37}$ (Fig. 1b). We trained the final ARA-CNN on 23,199 patches obtained after three

301 annotation and re-training iterations. We refer to this training dataset as LubLung (Materials and 
302 methods). The TME in the original slides differed between patients, which gave us a diverse set of

303 training examples. A range of tissue classes was selected individually by a pathologist and was used for

304 model training. Some of the slides were more covered by tumor and necrotic cells or stroma, while in

305 others immune infiltration, vessels or mixed class were dominating. In most of the slides we observed the

306 "normal" lung structures, so bronchi was less common and needed more training data from many sections

307 (Fig. 1c). The application of the final ARA-CNN on source LubLung tissue slides allowed to correctly

308 capture the TME heterogeneity in terms of all trained classes, which was confirmed by a pathologist who

309 compared the original H\&E slides with the final output of the model (Fig. 1d).

311 Next, we assessed the classification performance of ARA-CNN. To this end, we used a 10-fold cross-

312 validation procedure on the final set of 23,199 annotated patches obtained in the LubLung dataset

313 (Materials and methods). The best performance in a single class versus rest classification was achieved

314 for the background, lung, necrosis, tumor, and immune classes (area under the curve, AUC range: 0.97-

315 0.99) (Fig. 1e). The lowest AUC (0.83) was obtained for the mixed class, which is not surprising given

316 that it is a tissue that is a mix of two other classes (stroma and immune). We then computed a confusion

317 matrix, which confirmed that the best trained classes were background, necrosis, lung, immune and tumor

318 (accuracy range: 92.36\%-98.01) (Fig. 1f). In terms of errors, the model most often confused the mixed

319 class with tumor $(9.72 \%$ of the patches annotated as mixed were classified as tumor) or immune $(8.17 \%$

320 of the patches); the vessel class with stroma or lung (8.73\% and $10.79 \%$ of the patches, respectively); and

321 the bronchi class with tumor or lung $(7.30 \%$ and $8.53 \%$ of the patches, respectively). Given that patches

322 of these classes were also often hard to distinguish by an expert pathologist, we conclude that our trained

323 ARA-CNN model can reliably classify different tissue types in H\&E images of LUAD tissue sections.

\section{Identification of TME spatial composition features}

326 We then sought to apply our trained ARA-CNN model to study the spatial architecture of the TME in

327 H\&E images from 411 LUAD patients downloaded from the TCGA database (Supplementary Data). 
328 We split each image into $87 \times 87 \mu \mathrm{m}$ patches and then normalized each patch to the same color space as

329 the images in the LubLung dataset (Materials and methods). We used each patch as input to our ARA-

$330 \mathrm{CNN}$ model, which returned the probabilities of assigning each patch to one of the nine tissue classes. We

331 then segmented each image by assigning the most probable class to each patch. For each image, we

332 computed two metrics reflecting the spatial structure of the TME: TIP and TMEC ( Materials and

333 methods and Fig. 2a). TIP is represented by a vector of values $t_{i}$, computed as the fraction of patches

334 assigned to class $i$ out of all non-background patches in the whole slide image. TMEC is represented by a

335 vector of values $m_{i}$, computed as the fraction of patches assigned to class $i$ out of all non-tumor and non-

336 background tissue types in a predefined margin around the tumor tissue.

338 Across the investigated tissue classes, tumor and lung classes dominated the entire tissue composition,

339 with a median $t_{T U M O R}$ of 0.36 and a median $t_{L U N G}$ of 0.22 . The next three most abundant classes in the

340 LUAD slides were mixed, immune and bronchi (with median prevalence of around 0.07). Finally, the

341 least abundant classes were stroma, vessel, and necrosis. The most dominant classes of the tumor

342 microenvironment were lung (median $m_{L U N G}=0.32$ ), immune (median $\left.m_{I M M U N E}=0.18\right)$ and mixed

343 (median $m_{M I X E D}=0.17$ ). These classes were followed by bronchi (median $m_{B R O N C H I}=0.11$ ). The least

344 abundant in the tumor microenvironment were stroma, vessel and necrosis classes. This indicates that in

345 many patients, the tumor is surrounded by normal lung tissue and is confronted with an immune response.

346 The abundance of all features, however, showed large variability across the analyzed TCGA slides,

347 indicating high heterogeneity of both the entire tissue and the tumor microenvironment composition.

\section{TME features are predictive of patient survival}

350 We then explored if our metrics can be used to predict patient survival, given that the composition of the

351 TME has been previously shown to influence disease aggressiveness and survival in various cancer

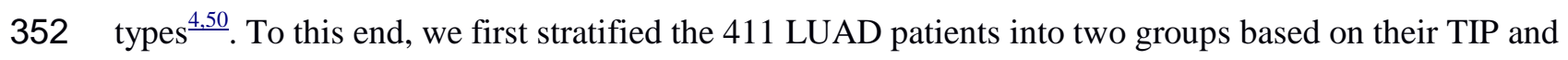

353 TMEC levels (High vs. Low). For each metric, we compared survival between the two groups using the 
354 Kaplan-Meier estimator (Materials and methods). Six TIP features (vessel $p=0.0016$, immune

$355 p=0.0058$, necrosis $p=0.0001$, stroma $p=0.0352$, bronchi $p=0.0079$ and mixed $p=0.0040)$ and five

356 TMEC features (vessel $p=0.0001$, immune $p=0.0045$, necrosis $p=0.0009$, stroma $p=0.0086$, and

357 bronchi $p=0.0254)$ showed statistically significant $(p<0.05, \log$ rank test, two-sided) differences in

358 survival between High and Low groups (Fig. 3a-k). To systematically assess the added value of the TIP

359 or TMEC and to compare them to other predictive features, we trained several versions of a multivariate

360 Cox proportional hazards model of the death hazard for the analyzed LUAD patients and assessed the

361 performance of each model with Harrell's c-index ${ }^{51}$ (Materials and methods). The best model yielded a

362 median c-index of 0.723 and included clinical data (age, sex, pathologic stage, and smoking status),

363 EGFR, STK11 and TP53 gene mutations, as well as TIP features, whereas inclusion of TMEC instead of

364 TIP features yielded a slightly lower c-index (0.709) (Fig. 31). All other models - including those based

365 on spatial diversity metrics such as Shannon index ${ }^{45}$, Simpson index ${ }^{44}$ and ITLR (Intra-Tumor

366 Lymphocyte Ratio $)^{\underline{21}}$ - resulted in lower c-index values. These results indicate that the TIP and TMEC

367 features, which respectively reflect the repertoire of different tissues and their proportions across the

368 entire examined tissue and across the TME,, are superior to other spatial metrics in predicting patient

369 survival.

370

371 Next, we assessed the association between TIP and TMEC features and the death hazard accounting for

372 the context of other features (Materials and methods). A hazard ratio of 1 for a given feature indicates

373 that the feature has no effect on survival, whereas a feature with hazard ratio larger than 1 indicates an

374 increased death hazard and, therefore, a negative impact on survival. The best performing model was

375 trained with clinical data, EGFR, STK11 and TP53 gene mutations, and TIP features. According to this

376 model, a high abundance of $t_{N E C R O S I S}$ and $t_{V E S S E L}$ features in the H\&E image was associated with

377 increased hazard. Similarly, abundance of $t_{B R O N C H I}$ and $t_{\text {STROMA }}$ features had a negative effect on

378 survival (Fig. 3m). In contrast, $t_{I M M U N E}$ and $t_{M I X E D}$ features were associated with a decreased death

379 hazard and therefore longer survival (Fig 3m), in line with the established role of the immune system as a 


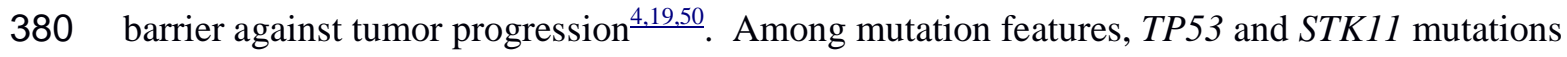

381 significantly increased $(p<0.05$, Wald test, two-sided) the death hazard, in agreement with the results of

382 the independent Kaplan-Meier analysis (Supplementary Fig. 1). A model trained with TMEC instead of

383 TIP features yielded very similar results (Fig. 3n). The impact of clinical features on the hazard agreed

384 with previously published results and our independent Kaplan-Meier analysis (Supplementary Results).

385

\section{TME features are predictive of disease-relevant mutations}

387 Next, we sought to investigate the association of the human-interpretable spatial composition features of

388 H\&E images with mutations in lung cancer genes. To this end, we trained classifiers for the mutation

389 status of 13 genes that are frequently mutated in LUAD: ALK, BRAF, DDR2, EGFR, KEAP1, KRAS,

390 MET, PDGFRB, PIK3CA, RET, ROS1, STK11, TP53. We evaluated eight different feature sets

391 (Materials and methods) with two machine learning algorithms: logistic regression and random forest.

392 Out of all 104 feature set and gene combinations, logistic regression was the better performing algorithm

393 in 55 cases, while random forest performed better in the remaining 47 cases, indicating that for some

394 genes non-linear relationships between the predictive features may be relevant for prediction of their

395 mutations (Table 1). For 8 out of 13 considered genes (namely, RET, KRAS, KEAP1, TP53, BRAF,

396 PDGFRB, ROS1, STK11), using the TIP or TMEC features gave the best result. For the remaining 5

397 genes (MET, ALK, DDR2, PIK3CA, EGFR), the best AUC was reached for models that utilized one of

398 previously existing spatial metrics as features.

399

400 The best AUC (73.5\%) was reached for the PDGFRB gene mutation by a classifier using clinical data and

401 both TIP and TMEC as features (Table 1). The best model without TIP and TMEC, and with the Simpson

402 metric as a feature, yielded an AUC smaller by 3.4 percentage points (p.p.). This shows that for the

403 PDGFRB gene mutation, the full information about tissue distribution, not reduced to a single value using

404 entropy and without focusing on only selected tissues, is highly relevant for its mutation status. The

405 classification performance of the best model using both TIP and TMEC for that gene is only slightly 
406

407

408

409

410

411

412 TMEC features, but including the Shannon metric, by around 9.1 p.p. Similarly, for KEAPl the

413 classification performance increased by 7 p.p. compared to models without TIP or TMEC. These results

414 indicate that, in LUAD, there exists a subset of tumor mutations that significantly correlate with how the

415 TME is structured, and that both TIP and TMEC features are predictive of the presence of these

416 mutations.

417

418 We then inspected the two best performing models in the mutation classification task that utilised TIP and

419 TMEC features to find which predictor features were the most important for identifying mutations. Both

420

421

422

423

424

425

426

427

428

429

430

431 of the algorithms used - logistic regression and random forest - are easily interpretable because they allow effective identification of the most important features. First, we analysed the logistic regression classifier of PDGFRB mutations with clinical, TMEC and TIP features (Fig. 4a). The most important features positively correlated with $P D G F R B$ mutation were sex, $m_{M I X E D}$ - corresponding to the proportion of the mixed tissue in the tumor microenvironment - and $t_{T U M O R}-$ corresponding to the fraction of the entire slide occupied by the tumor. On the other hand, the most negatively correlated (i.e., decreasing the chance of mutation) features were non-smoker status, $t_{I M M U N E}$, and $m_{B R O N C H I}$. Next, we inspected the random forest classifier of RET mutations, which included clinical and TMEC features in its feature set (Fig. 4b). The latter proved to be of larger importance than the former ones. Indeed, RET mutations were found to be most associated with the prevalence of different tissues in the tumor microenvironment, with bronchi and vessels identified as the most impactful tissues, followed by mixed, stroma, lung, immune and necrosis. This observation might be explained by the fact that, in LUAD, RET mutations mainly 
432

433

434

435

436

437

438

439

440

441

442

443

444

445

\section{Discussion}

447 We have developed a novel H\&E image classification model, ARA-CNN, and a training dataset of 448 annotated tissue patches from LUAD H\&E images, LubLung. Both considerably expand the current

449 ability to analyze the TME automatically and quantitatively in lung cancer samples, which in turn has 450 important implications for patient stratification and precision treatment. TIP and TMEC metrics, which 451 we have introduced in this work, provide a novel way of capturing the composition and spatial structure 452 of the TME, and are predictive of both overall survival and clinically relevant mutations. Spatial statistics 453 of H\&E images in the form of metrics that quantify colocalization of cell or tissue types, have been

454 previously shown to be predictive of patient survival ${ }^{21}$. However, these metrics are computed based on a 455 limited number of features, such as counts of tumor and immune cells. Other approaches that try to link 
456 the structure of tumor tissue and TME with either gene mutations or patient survival are end-to-end deep

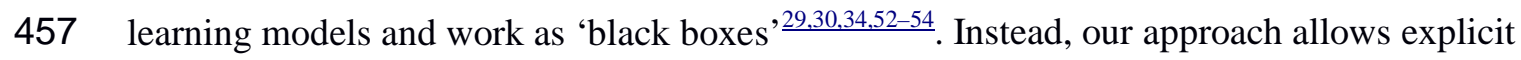

458 interpretability, as it decouples H\&E slide inference from downstream tasks (e.g., mutation classification

459 and survival analysis). The TIP and TMEC features are per se human interpretable, so it is possible to

460 precisely pinpoint which tissue types are the most important. Our approach requires the initial tissue

461 classification to be as accurate as possible. We ensured this to be the case by using ARA-CNN, which

462 performs excellently in classifying nine tissue classes present in lung cancer H\&E images. To foster

463 further research in predictive spatial statistics based on a rich repertoire of segmented lung cancer tissues,

464 in addition to LubLung we also share the segmented TCGA images as a separate dataset, named

465 SegLungTCGA.

466

467 Our analysis revealed that patient stratification based on TIP and TMEC features yields significant

468 differences in patient survival between the strata. Moreover, the most predictive survival models included

469 TIP and TMEC features. These findings are supported by previous clinical studies. It has been shown that

470 blood vessel invasion is a major prognostic factor in lung cancer survival ${ }^{14,55}$. Similarly, there have been

471 studies which proved that tumor necrosis is a significant risk factor for survival in lung cancer ${ }^{56}$.

472 However, the complexity of the entire lung microenvironment plays a key role in the development of

473 primary lung carcinomas and offers a resource of targets for personalized therapy development. Targeting

474 the angiogenesis and immune cells has elucidated the prognostic and pathophysiological roles of other

475 components of the TME in lung cancer ${ }^{13,57}$. In the end, the combination of the clinical and genetic

476 information with the TME landscape may play a pivotal role in predicting the type and duration of

477 response to personalized therapies.

478

479 We found eight genes relevant to lung cancer (PDGFRB, RET, KRAS, KEAP1, ROS1, STK11, MET and

$480 A L K$ ), for which integrating clinical data with our TME metrics clearly improves the ability to predict

481 mutations in these genes. We speculate that mutations of these genes may alter cellular interactions, and 
482 hence the spatial arrangement of the TME visible in H\&E images. For RET, ROS1 and ALK genes,

483 mutations mainly consist of chromosomal rearrangements which produce chimeric proteins that might

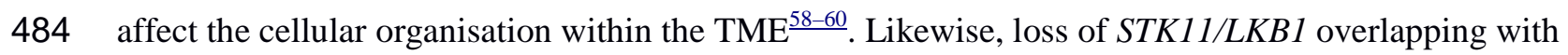

485 oncogenic KRAS mutations is associated with increased neutrophil recruitment, and decreased T-cells

486 infiltration in lung cancer tumors ${ }^{61}$. Moreover, STK11 mutations often coexist with KEAP1 mutations that

487 relate to cellular resistance to oxidative stress $\frac{62}{}$, and co-occurrence of KEAPl mutations and PTEN

488 inactivation is an indicator of an immunologically "cold" tumor ${ }^{63}$. We speculate that each of these

489 mutations might slightly affect the cellular morphology in H\&E images in a way that is not apparent to

490 the human eye, but can be captured by deep-learning algorithms.

491

492 Our findings concern mutations of clinically relevant genes, and as such may have clinical implications.

493 For example, both RET and PDGFRB are clinically relevant LUAD cancer genes. RET has proto-

494 oncogene properties and its fusions, which occur in $1-2 \%$ of $\operatorname{LUAD}^{64}$, are associated with a high risk of

495 brain metastasis ${ }^{65}$. However, last clinical trials indicated that they may be effectively targeted by RET

496 tyrosine kinase inhibitors as pralsetinib, selpercatinib ${ }^{64}$. PDGFRB is a member of the PDGF/PDGFR axis

497 that is recognized as a key regulator of mesenchymal cell activity in $\mathrm{TME}^{\frac{66}{}}$, and several new agents

498 (linifanib, motesanib, olaratumab) that block the PDGFR signaling are being tested in LUAD ${ }^{67}$. In breast,

499 colon, pancreas and prostate cancers, the high stromal expression of the PDGFR $\beta$ protein has been

500 associated with poor prognosis $\frac{67}{}$, however its prognostic relevance in tumors of epithelial origin is

501 inconclusive $e^{\frac{66}{}}$. It was only confirmed that a relative expression of PDGFRs is a strong and independent

502 predictor of longer survival for surgical stages of lung cancer (I-IIIA) ${ }^{67}$.

503

504 Our approach has several limitations. In the mutation classification tasks, we used simple machine

505 learning models - logistic regression and random forest. An end-to-end deep learning model might give

506 better results, however, as discussed above, these models suffer when it comes to interpretability. Another

507 limitation is the fact that ARA-CNN works on a patch-based basis. An alternative to that is a cell-based 
508 classifier, which could produce more fine-grained segmentations and in turn enable a more precise

509 computation of spatial statistics. On the other hand, with a patch-based approach, a suitably small patch

510 size can be selected, as we did in this study. Such small patches can be assumed to be homogeneous when

511 it comes to cell types and can enable a precise computation of summary statistics such as the TIP and

512 TMEC metrics, that we have introduced here. Furthermore, in the survival prediction task we did not have

513 access to and hence did not utilise the treatment information as features. Since treatment has a large

514 impact on survival, using this data is expected to improve prediction performance by a large margin.

515 However, the main focus of our study was not to deliver the best performing survival prediction approach,

516 but rather to assess the predictive power of our proposed spatial composition features, which can be

517 performed without including the treatment data in the model. Lastly, to apply our approach to another

518 cancer type, one would need to retrain the ARA-CNN model, which necessitates substantial input from a

519 trained pathologist. This training effort can be minimised by utilising the active learning component of

520 ARA, which shortens the number of iterations required to build an effective training dataset. For

521 colorectal cancer, a pre-trained model is available from a previous study ${ }^{37}$.

522

523 The analysis presented here shows that there is a correspondence between the spatial structure in $\mathrm{H} \& \mathrm{E}$

524 images for LUAD and both gene mutations and patient survival. Not every mutation is expected to have

525 an effect on tissue prevalence or tumor neighbourhood structure, so it is not surprising that for some of

526 the analyzed genes the mutation classification performance did not exceed an AUC of 0.6. In contrast, it

527 is striking that there are genes for which adding tissue composition data to the clinical information

528 improves classification results. Finally, it is also surprising that our TIP and TMEC metrics, as well as

529 other metrics of TME spatial organization, such as ITLR, can give good results in terms of both mutation

530 classification and survival analysis.

531

532 We have explored the applicability of human interpretable features extracted from deep learning-based

533 segmentation of H\&E images. Our approach can provide important insights for designing novel cancer 
534 treatments, by linking the spatial structure of the tumor microenvironment in LUAD to gene mutations

535 and patient survival. It can also expand our understanding of the effects that the tumor microenvironment

536 has on tumor evolutionary processes. We therefore envision that, in the future, our quantitative approach

537 will become incorporated in routine diagnostics for LUAD and other cancer types.

\section{Data availability}

539 The LubLung dataset is available publically at github.com/animgoeth/LubLung

540 The SegLungTCGA dataset is available at github.com/animgoeth/SegLungTCGA

541 The ARA-CNN weights trained on LubLung are available at github.com/animgoeth/ARA-CNN, in the

542 'pretrained' directory.

543 The Supplementary Data is available per authors' discretion.

\section{References}

545 1. Fox, H. Is H\&E morphology coming to an end? J. Clin. Pathol. 53, 38-40 (2000).

546 2. Komura, D. \& Ishikawa, S. Machine Learning Methods for Histopathological Image Analysis.

547 Comput. Struct. Biotechnol. J. 16, 34-42 (2018).

548 3. Djuric, U., Zadeh, G., Aldape, K. \& Diamandis, P. Precision histology: how deep learning is

549 poised to revitalize histomorphology for personalized cancer care. Npj Precis. Oncol. 1, 1-5

$550 \quad$ (2017).

551 4. Binnewies, M. et al. Understanding the tumor immune microenvironment (TIME) for effective

552 therapy. Nat. Med. 24, 541-550 (2018).

553 5. Griffiths, A. J. et al. An Introduction to Genetic Analysis. (W. H. Freeman, 2000).

554 6. Abedi, S. et al. Estimating the Survival of Patients With Lung Cancer: What Is the Best

$555 \quad$ Statistical Model? J. Prev. Med. Pub. Health 52, 140-144 (2019).

556 7. Hassan, M. R. A. et al. Survival Analysis and Prognostic Factors for Colorectal Cancer 
$557 \quad$ Patients in Malaysia. Asian Pac. J. Cancer Prev. APJCP 17, 3575-3581 (2016).

558 8. Anderson, N. M. \& Simon, M. C. The tumor microenvironment. Curr. Biol. 30, R921-R925

$559 \quad \underline{(2020)}$.

560 9. Bade, B. C. \& Dela Cruz, C. S. Lung Cancer 2020: Epidemiology, Etiology, and Prevention.

$561 \quad$ Clin. Chest Med. 41, 1-24 (2020).

562 10. Ruiz-Cordero, R. \& Devine, W. P. Targeted Therapy and Checkpoint Immunotherapy in

$563 \quad$ Lung Cancer. Surg. Pathol. Clin. 13, 17-33 (2020).

564 11. Imyanitov, E. N., lyevleva, A. G. \& Levchenko, E. V. Molecular testing and targeted therapy

565 for non-small cell lung cancer: Current status and perspectives. Crit. Rev. Oncol. Hematol.

$566 \quad \underline{157,103194(2021)}$.

567 12. Duma, N., Santana-Davila, R. \& Molina, J. R. Non-Small Cell Lung Cancer: Epidemiology,

568 Screening, Diagnosis, and Treatment. Mayo Clin. Proc. 94, 1623-1640 (2019).

569 13. Altorki, N. K. et al. The lung microenvironment: an important regulator of tumour growth and

$570 \quad$ metastasis. Nat. Rev. Cancer 19, 9-31 (2019).

571 14. Zhang, C., Liu, Y., Guo, S. \& Zhang, J. Different biomarkers in non-small cell lung cancer in

572 blood vessel invasion. Cell Biochem. Biophys. 70, 777-784 (2014).

573 15. Chan, T. A. et al. Development of tumor mutation burden as an immunotherapy biomarker:

$574 \quad$ utility for the oncology clinic. Ann. Oncol. 30, 44-56 (2019).

575 16. Rehman, J. A. et al. Quantitative and pathologist-read comparison of the heterogeneity of

576 programmed death-ligand 1 (PD-L1) expression in non-small cell lung cancer. Mod. Pathol.

$577 \quad 30,340-349(2017)$.

578 17. Huang, Y.-K. et al. Macrophage spatial heterogeneity in gastric cancer defined by multiplex

579 immunohistochemistry. Nat. Commun. 10, 3928 (2019).

580 18. Heindl, A. et al. Relevance of Spatial Heterogeneity of Immune Infiltration for Predicting Risk

581 of Recurrence After Endocrine Therapy of ER+ Breast Cancer. JNCl J. Natl. Cancer Inst.

$582 \quad \underline{110,166-175(2018)}$. 
583 19. Rudolf, J. et al. Regulatory T cells and cytotoxic T cells close to the epithelial-stromal

$584 \quad$ interface are associated with a favorable prognosis. Oncolmmunology 9, 1746149 (2020).

585 20. Valous, N. A., Moraleda, R. R., Jäger, D., Zörnig, I. \& Halama, N. Interrogating the

$586 \quad$ microenvironmental landscape of tumors with computational image analysis approaches.

$587 \quad$ Semin. Immunol. 48, 101411 (2020).

588 21. Yuan, Y. Modelling the spatial heterogeneity and molecular correlates of lymphocytic

$589 \quad$ infiltration in triple-negative breast cancer. J. R. Soc. Interface 12, 20141153 (2015).

590 22. Saltz, J. Spatial Organization and Molecular Correlation of Tumor-Infiltrating Lymphocytes

$591 \quad$ Using Deep Learning on Pathology Images. 21 (2018).

592 23. Yuan, Y. Spatial Heterogeneity in the Tumor Microenvironment. Cold Spring Harb. Perspect.

$593 \quad$ Med. 6, a026583 (2016).

594 24. Heindl, A., Lan, C., Rodrigues, D. N., Koelble, K. \& Yuan, Y. Similarity and diversity of the

595 tumor microenvironment in multiple metastases: critical implications for overall and

596 progression-free survival of high-grade serous ovarian cancer. Oncotarget 7, 71123-71135

$597 \quad \underline{(2016)}$.

598 25. Corredor, G. et al. Spatial Architecture and Arrangement of Tumor-Infiltrating Lymphocytes

599 for Predicting Likelihood of Recurrence in Early-Stage Non-Small Cell Lung Cancer. Clin.

$600 \quad$ Cancer Res. 25, 1526-1534 (2019).

601 26. Xi, K.-X. et al. Tumor-stroma ratio (TSR) in non-small cell lung cancer (NSCLC) patients

602 after lung resection is a prognostic factor for survival. J. Thorac. Dis. 9, 4017-4026 (2017).

603 27. Mobadersany, P. et al. Predicting cancer outcomes from histology and genomics using

$604 \quad$ convolutional networks. Med. Sci. 10 (2018).

605 28. Yousefi, S. et al. Predicting clinical outcomes from large scale cancer genomic profiles with

$606 \quad$ deep survival models. Sci. Rep. 7, 11707 (2017).

607 29. Chen, M. et al. Classification and mutation prediction based on histopathology H\&E images

$608 \quad$ in liver cancer using deep learning. Npj Precis. Oncol. 4, 1-7 (2020). 
bioRxiv preprint doi: https://doi.org/10.1101/2021.10.09.462574: this version posted October 92021 . The copyright holder for this preprint (which was not certified by peer review) is the author/funder, who has granted bioRxiv a license to display the preprint in perpetuity. It is made available under aCC-BY-NC 4.0 International license.

609 30. Liao, H. et al. Deep learning $\square$ based classification and mutation prediction from

610 histopathological images of hepatocellular carcinoma. Clin. Transl. Med. 10, (2020).

611 31. Chen, X., Lin, X., Shen, Q. \& Qian, X. Combined Spiral Transformation and Model-Driven

612 Multi-Modal Deep Learning Scheme for Automatic Prediction of TP53 Mutation in Pancreatic

$613 \quad$ Cancer. IEEE Trans. Med. Imaging 40, 735-747 (2021).

614 32. Wu, Z., Huang, X., Huang, S., Ding, X. \& Wang, L. Direct Prediction of BRAFV600E

615 Mutation from Histopathological Images in Papillary Thyroid Carcinoma with a Deep Learning

$616 \quad$ Workflow. in 2020 4th International Conference on Computer Science and Artificial

617 Intelligence 146-151 (Association for Computing Machinery, 2020).

$618 \quad$ doi: $10.1145 / 3445815.3445840$.

619 33. Schaumberg, A. J., Rubin, M. A. \& Fuchs, T. J. H\&E-stained Whole Slide Image Deep

620 Learning Predicts SPOP Mutation State in Prostate Cancer.

621 http://biorxiv.org/lookup/doi/10.1101/064279 (2016) doi:10.1101/064279.

622 34. Kather, J. N. et al. Pan-cancer image-based detection of clinically actionable genetic

623 alterations. Nat. Cancer 1, 789-799 (2020).

624 35. Diao, J. A. et al. Human-interpretable image features derived from densely mapped cancer

625 pathology slides predict diverse molecular phenotypes. Nat. Commun. 12, 1613 (2021).

626 36. Bankhead, P. et al. QuPath: Open source software for digital pathology image analysis. Sci.

$627 \quad$ Rep. 7, 16878 (2017).

628 37. Raczkowski, Ł., Możejko, M., Zambonelli, J. \& Szczurek, E. ARA: accurate, reliable and

629 active histopathological image classification framework with Bayesian deep learning. Sci.

$630 \quad$ Rep. 9, $14347(2019)$.

631 38. He, K., Zhang, X., Ren, S. \& Sun, J. Deep Residual Learning for Image Recognition.

$632 \quad$ ArXiv151203385 Cs (2015).

633 39. Redmon, J. \& Farhadi, A. YOLO9000: Better, Faster, Stronger. in 2017 IEEE Conference on

634 Computer Vision and Pattern Recognition (CVPR) 6517-6525 (2017). 
doi:10.1109/CVPR.2017.690.

636 40. loffe, S. \& Szegedy, C. Batch Normalization: Accelerating Deep Network Training by

$637 \quad$ Reducing Internal Covariate Shift. in International Conference on Machine Learning 448-456

$638 \quad$ (PMLR, 2015).

639 41. Srivastava, N., Hinton, G., Krizhevsky, A., Sutskever, I. \& Salakhutdinov, R. Dropout: A

$640 \quad$ Simple Way to Prevent Neural Networks from Overfitting. J. Mach. Learn. Res. 15, 1929-

$641 \quad \underline{1958(2014) .}$

642 42. Gal, Y. \& Ghahramani, Z. Bayesian Convolutional Neural Networks with Bernoulli

$643 \quad$ Approximate Variational Inference. ArXiv150602158 Cs Stat (2016).

644 43. Kingma, D. P. \& Ba, J. Adam: A Method for Stochastic Optimization. ArXiv14126980 Cs

$645 \quad$ (2017).

646 44. Simpson, E. H. Measurement of Diversity. Nature 163, 688-688 (1949).

647 45. Shannon, C. E. A mathematical theory of communication. ACM SIGMOBILE Mob. Comput.

$648 \quad$ Commun. Rev. 5, 3-55 (2001).

649 46. Fan, R.-E., Chang, K.-W., Hsieh, C.-J., Wang, X.-R. \& Lin, C.-J. LIBLINEAR: A Library for

$650 \quad$ Large Linear Classification. J. Mach. Learn. Res. 9, 1871-1874 (2008).

651 47. Breiman, L. Random Forests. Mach. Learn. 45, 5-32 (2001).

652 48. Pedregosa, F. et al. Scikit-learn: Machine Learning in Python. J. Mach. Learn. Res. 12.

$653 \quad 2825-2830(2011)$.

654 49. Ramos, M. curatedTCGAData: Curated Data From The Cancer Genome Atlas (TCGA) as

$655 \quad$ MultiAssayExperiment Objects. (Bioconductor, 2020).

656 doi:10.18129/B9.BIOC.CURATEDTCGADATA.

657 50. Oliver, A. J. et al. Tissue-Dependent Tumor Microenvironments and Their Impact on

$658 \quad$ Immunotherapy Responses. Front. Immunol. 9, 70 (2018).

659 51. Harrell, F. E., Jr, Califf, R. M., Pryor, D. B., Lee, K. L. \& Rosati, R. A. Evaluating the Yield of

$660 \quad$ Medical Tests. JAMA 247, 2543-2546 (1982). 
bioRxiv preprint doi: https://doi org/10.1101/2021.10.09.462574: this version posted October 92021 . The copyright holder for this preprint (which was not certified by peer review) is the author/funder, who has granted bioRxiv a license to display the preprint in perpetuity. It is made available under aCC-BY-NC 4.0 International license.

661 52. Coudray, N. et al. Classification and mutation prediction from non-small cell lung cancer

662 histopathology images using deep learning. Nat. Med. 24, 1559-1567 (2018).

663 53. Bian, C. et al. ImmunoAlzer: A Deep Learning-Based Computational Framework to

664 Characterize Cell Distribution and Gene Mutation in Tumor Microenvironment. Cancers 13,

$665 \quad \underline{1659(2021) .}$

666 54. Courtiol, P. et al. Deep learning-based classification of mesothelioma improves prediction of

667 patient outcome. Nat. Med. 25, 1519-1525 (2019).

668 55. Kessler, R. et al. Blood vessel invasion is a major prognostic factor in resected non-small

669 cell lung cancer. Ann. Thorac. Surg. 62, 1489-1493 (1996).

670 56. Park, S. Y. et al. Tumor Necrosis as a Prognostic Factor for Stage IA Non-Small Cell Lung

671 Cancer. Ann. Thorac. Surg. 91, 1668-1673 (2011).

672 57. Chen, Z., Fillmore, C. M., Hammerman, P. S., Kim, C. F. \& Wong, K.-K. Non-small-cell lung

673 cancers: a heterogeneous set of diseases. Nat. Rev. Cancer 14, 535-546 (2014).

674 58. Sholl, L. M. et al. ROS1 immunohistochemistry for detection of ROS1-rearranged lung

675 adenocarcinomas. Am. J. Surg. Pathol. 37, 1441-1449 (2013).

676 59. Wang, R. et al. RET fusions define a unique molecular and clinicopathologic subtype of non-

677 small-cell lung cancer. J. Clin. Oncol. Off. J. Am. Soc. Clin. Oncol. 30, 4352-4359 (2012).

678 60. Nishino, M. et al. Histologic and cytomorphologic features of ALK-rearranged lung

679 adenocarcinomas. Mod. Pathol. Off. J. U. S. Can. Acad. Pathol. Inc 25, 1462-1472 (2012).

680 61. Skoulidis, F. et al. STK11/LKB1 Mutations and PD-1 Inhibitor Resistance in KRAS-Mutant

$681 \quad$ Lung Adenocarcinoma. Cancer Discov. 8, 822-835 (2018).

682 62. Papillon-Cavanagh, S., Doshi, P., Dobrin, R., Szustakowski, J. \& Walsh, A. M. STK11 and

$683 \quad$ KEAP1 mutations as prognostic biomarkers in an observational real-world lung

684 adenocarcinoma cohort. ESMO Open 5, e000706 (2020).

685 63. Błach, J., Wojas-Krawczyk, K., Nicoś, M. \& Krawczyk, P. Failure of Immunotherapy-The

686 Molecular and Immunological Origin of Immunotherapy Resistance in Lung Cancer. Int. J. 
Mol. Sci. 22, 9030 (2021).

688 64. Drusbosky, L. M., Rodriguez, E., Dawar, R. \& Ikpeazu, C. V. Therapeutic strategies in RET

689 gene rearranged non-small cell lung cancer. J. Hematol. Oncol.J Hematol Oncol 14, 50

$690 \quad$ (2021).

691 65. Drilon, A. et al. Frequency of Brain Metastases and Multikinase Inhibitor Outcomes in

692 Patients With RET-Rearranged Lung Cancers. J. Thorac. Oncol. 13, 1595-1601 (2018).

693 66. Paulsson, J., Ehnman, M. \& Östman, A. PDGF receptors in tumor biology: prognostic and

694 predictive potential. Future Oncol. Lond. Engl. 10, 1695-1708 (2014).

695 67. Kilvaer, T. K. et al. Differential prognostic impact of platelet-derived growth factor receptor

696 expression in NSCLC. Sci. Rep. 9, 10163 (2019).

697

698 Funding

699 This work was supported by a Mobilnosc Plus scholarship from the Polish Ministry of Science and High

700 Education (1622/MOB/V/2017/0) and a grant from the Polish National Science Center (UMO-

701 2016/23/D/NZ2/02890) to M.N.; by grants from the Swedish Research Council (521-2014-2866), the

702 Swedish Cancer Research Foundation (CAN 2015/585), the Ragnar Söderberg Foundation, the Swedish

703 Foundation for Strategic Research (BD15-0095), and the Strategic Research Programme in Cancer

704 (StratCan) at Karolinska Institutet to N.C.; and by OPUS grant no. 2019/33/B/NZ2/00956 to ES from the

705 National Science Centre, Poland, https://www.ncn.gov.pl/?language=en.

\section{Author contributions}

707 Ł.R. performed all experiments, analysed the results and prepared visualisations. I.P. was responsible for

708 H\&E image annotation and segmentation validation. Ł.K., I.P., M.N. and E.S. coordinated the active

709 learning process. M.K. contributed to the work on survival analysis. M.B. provided code for extracting

710 mutation data from TCGA. M.N. and P.K. helped with the interpretation of clinical data and provided 
711 feedback on the results. J.S. provided the access for the archival tissue material used for the model

712 training. M.N collected the material and prepared the tissue sections. T.K performed the H\&E staining

713 and scanned all the slides used for the model training. E.S. and N.C. supervised the research. Ł.R. and

714 E.S. wrote the manuscript. Ł.R., E.S., M.N. and N.C. conceptualised the project. All authors reviewed the

715 manuscript.

\section{Corresponding author}

717 Correspondence should be addressed to Ewa Szczurek.

a

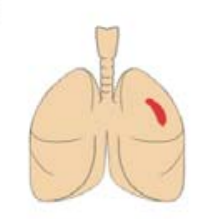

55 lung cancer patients
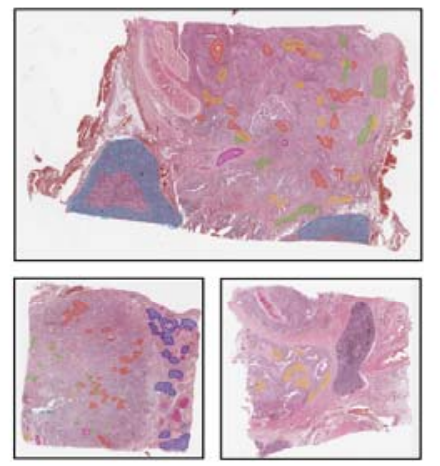

b
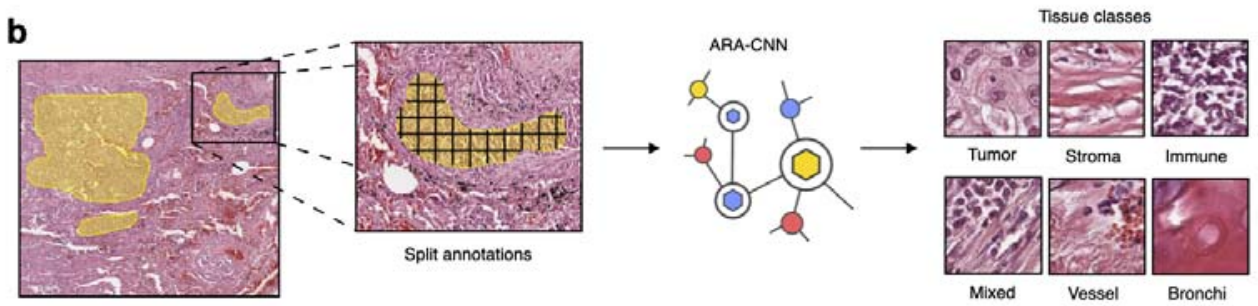

Annotated slide

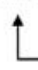

d

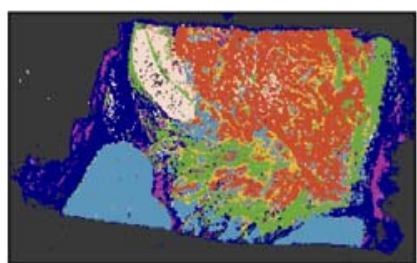

Pathologist

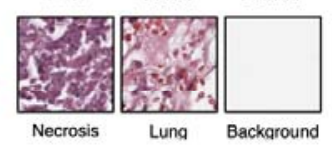

e
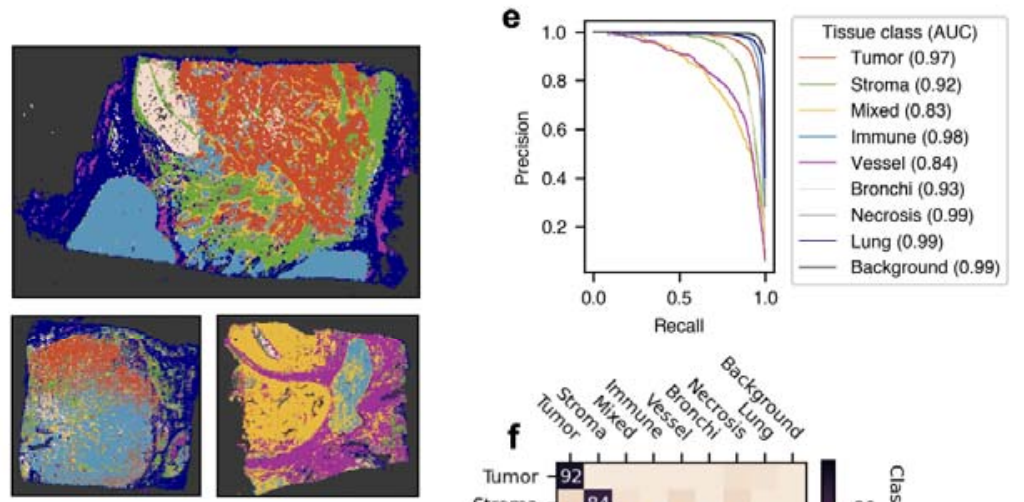

E Tumor $=$ Stroma

IIIxed Vessel

Necrosis Lung

inmune Bronchi

Background

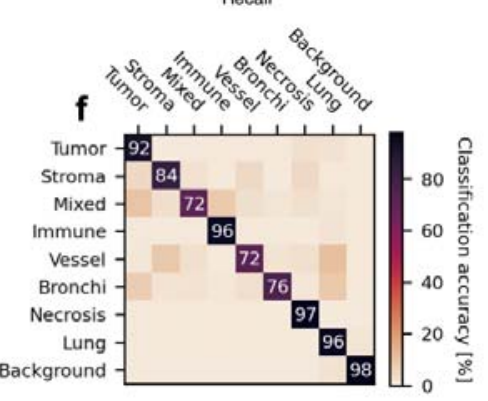

719 Figure 1. Overview of training ARA-CNN for lung cancer tissue classification (a) We sourced H\&E 
720 tissue slides from 55 lung cancer patients (b) These slides were annotated by an expert pathologist in an

721 active learning loop with ARA-CNN, which resulted in the LubLung dataset and a trained tissue

722 classification model. (c) Example annotations of various tissue regions (d) Segmentation results from

723 ARA-CNN show that tissue heterogeneity in the TME is captured correctly (e) Precision-recall curves for 724 each tissue class obtained in a 10-fold cross-validation scheme on the LubLung dataset. The mean AUC is 725 0.94. (f) Confusion matrix for ARA-CNN trained with LubLung. Row labels indicate true classes, while 726 column labels describe classes predicted by the model.

a
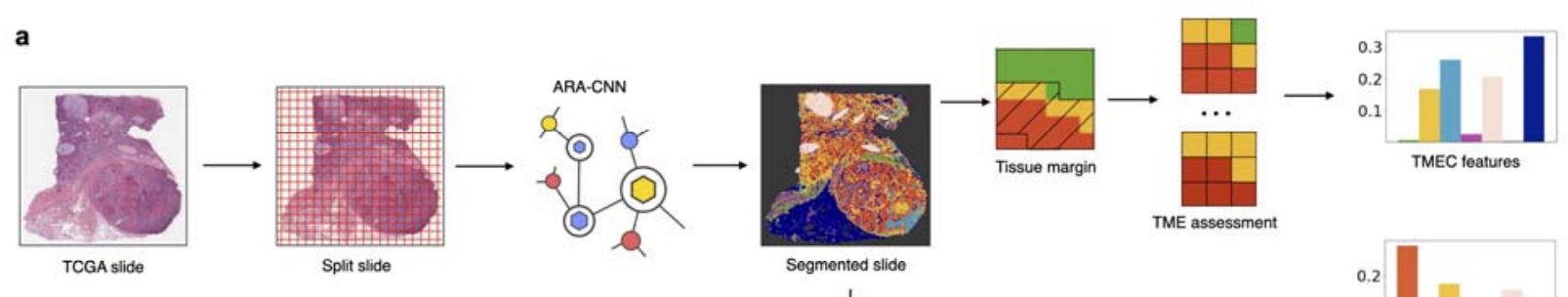

Segmented slide

0.2

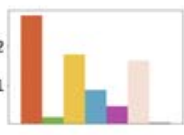

b

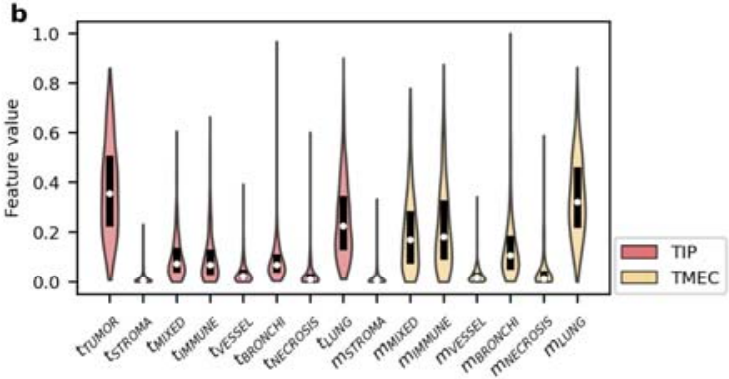

c

TIP features
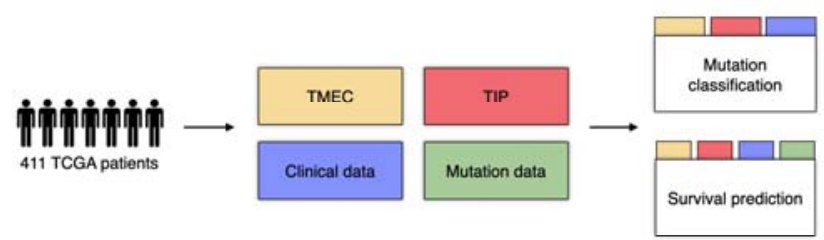

729

Figure 2. Calculation and utilisation of TIP and TMEC features (a) H\&E slides from TCGA were

731 downloaded and split into tissue patches. Each patch was classified with ARA-CNN, producing tissue

732 segmentations. These segmentations were next used to calculate the TIP and TMEC features. (b)

733 Distribution of individual component features in TIP and TMEC. The most often occurring features for

734 TIP were $t_{T U M O R}$ and $t_{L U N G}$. For TMEC, these were $m_{L U N G}, m_{I M M U N E}$ and $m_{M I X E D}$. (c) Tasks performed with

735 the help of the TIP and TMEC features. In addition to the TIP and TMEC features, clinical and mutation

736 data was also sourced from TCGA. These datasets were combined and served as input in two tasks: 
bioRxiv preprint doi: https://doi.org/10.1101/2021.10.09.462574; this version posted October 9, 2021. The copyright holder for this preprint

(which was not certified by peer review) is the author/funder, who has granted bioRxiv a license to display the preprint in perpetuity. It is made available under aCC-BY-NC 4.0 International license.

737 survival prediction and gene mutation classification. The results were compared to those obtained using

738 previous spatial metrics instead of TIP and TMEC.

739

740

741

742

743

744 

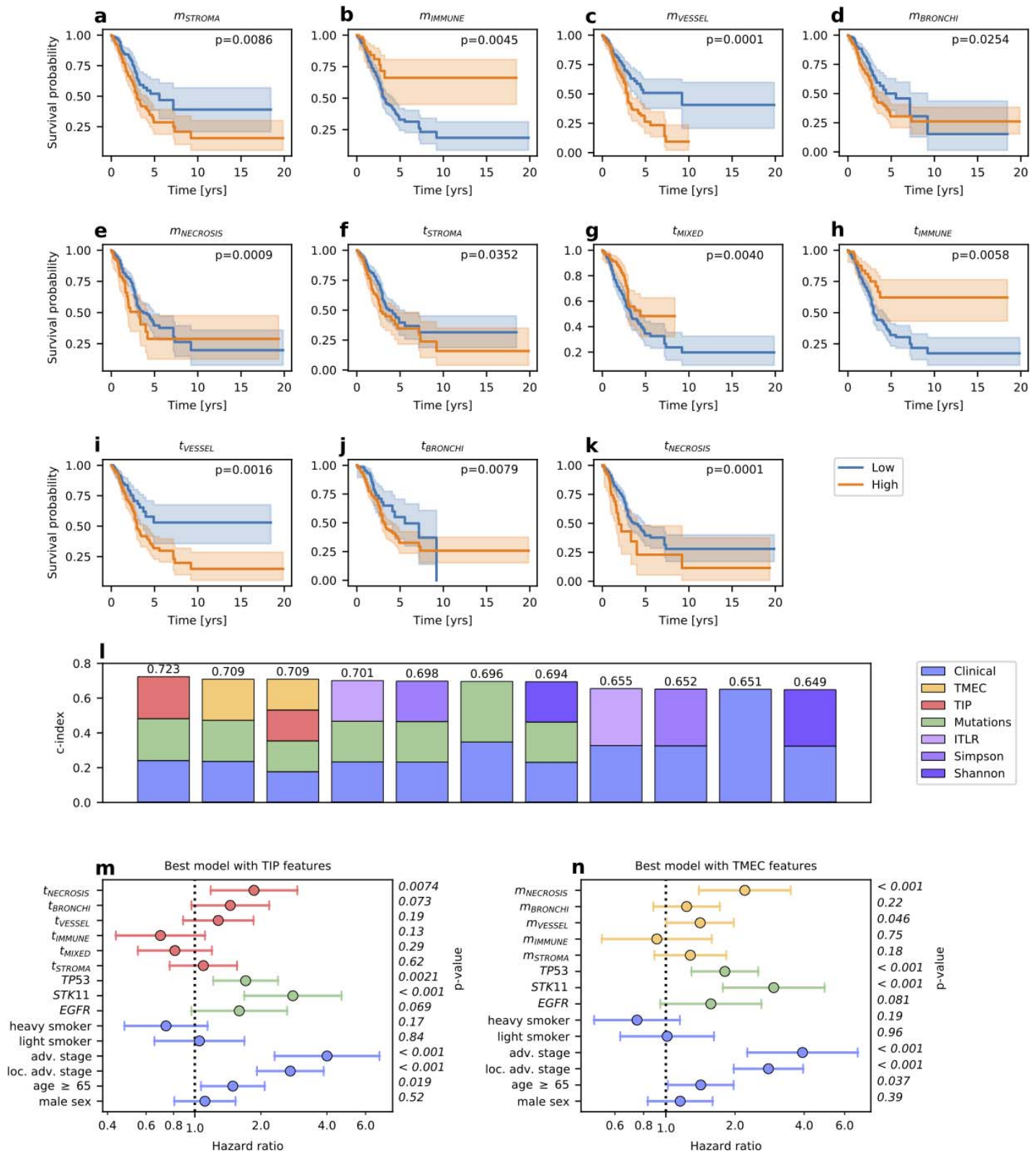

745

746 Figure 3. Survival prediction results. (a-k) Kaplan-Meier plots for TIP and TMEC features that result in

747 patient stratification into two groups: with high and low values of the feature. Only features with

748 statistically significant differences in patient survival are shown, as measured using the log rank test (p-

749 values in the top right corner). The results correlate with previous studies of the relationship between

750 these features and patient survival. (l) c-index scores for Cox models from survival prediction

751 experiments performed with different feature sets. The best results were obtained for models with such 
752 feature sets that included TIP and TMEC features. (m) Hazard ratios for the best model that utilised the

753 TIP features. The prevalence of the necrosis tissue class in the whole slide has a statistically significant

754 negative effect on survival. (n) Hazard ratios for the best model that utilised the TMEC features. The

755 presence of the necrosis tissue class and the vessel tissue class in the TME has a statistically significant

756 negative effect on survival.

757

758

759

760

761

762

763

764

765

766

767

768

769

770

771

772

773

774 

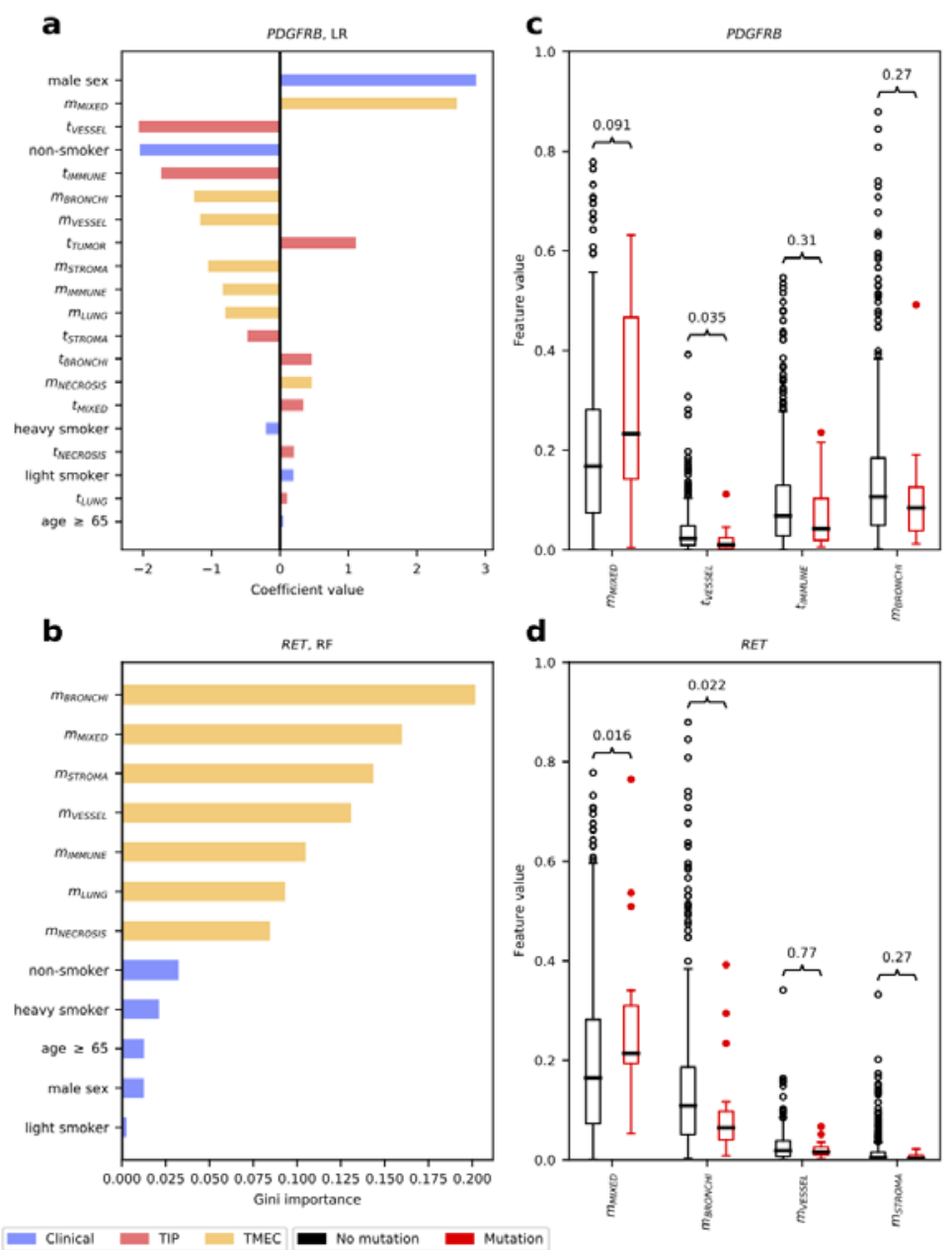

776 Figure 4. Feature importance for the two best performing mutation classification models that utilised TIP

777 and TMEC features (a) Feature importance for the PDGFRB gene mutation classifier (logistic

778 regression). Here, feature importance is measured by the value of its regression coefficient. (b) Feature

779 importance for the RET gene mutation classifier (random forest). Here, the importance is measured by the

780 reduction of the Gini index obtained when the feature is added to the tree, averaged across the trees in the

781 random forest model. (c) Distribution of feature values for four of the most important TIP or TMEC

782 features, as presented in (a), divided between patients with the mutated and non-mutated PDGFRB gene.

783 (d) Distribution of feature values for four of the most important TMEC features, as presented in (b),

784 divided between patients with the mutated and non-mutated RET gene. 
786 Table 1. Mutation/rearrangement classification AUC scores (given as \% of area under the precision-recall

787 curve) for TCGA LUAD patients. The best result for each gene is marked in bold. In cases where the

788 random forest classifier gave the best result, the cells are colored in yellow. Otherwise, if logistic

789 regression gave the best result, the cells are colored in light blue.

790

\begin{tabular}{|c|c|c|c|c|c|c|c|c|c|}
\hline Gene & $\begin{array}{c}\text { Mutation } \\
\text { count }\end{array}$ & Clinical & $\begin{array}{c}\text { Clinical + } \\
\text { ITLR }\end{array}$ & $\begin{array}{l}\text { Clinical + } \\
\text { Shannon }\end{array}$ & $\begin{array}{c}\text { Clinical + } \\
\text { Simpson }\end{array}$ & $\begin{array}{c}\text { Clinical + } \\
\text { TMEC }\end{array}$ & $\begin{array}{c}\text { Clinical + } \\
\text { TIP }\end{array}$ & $\begin{array}{c}\text { Clinical + } \\
\text { TMEC + } \\
\text { TIP }\end{array}$ & TMEC \\
\hline$R E T$ & 25 & $\begin{array}{c}53.70 \pm \\
2.21\end{array}$ & $\begin{array}{c}50.76 \pm \\
2.39\end{array}$ & $\begin{array}{c}58.03 \pm \\
2.13\end{array}$ & $\begin{array}{c}57.76 \pm \\
2.09\end{array}$ & $\begin{array}{c}67.14 \pm \\
2.75\end{array}$ & $\begin{array}{c}59.46 \pm \\
2.13\end{array}$ & $\begin{array}{c}65.32 \pm \\
1.95\end{array}$ & $\begin{array}{c}64.39 \pm \\
2.73\end{array}$ \\
\hline$K R A S$ & 96 & $\begin{array}{c}61.12 \pm \\
0.88\end{array}$ & $\begin{array}{c}61.36 \pm \\
0.84\end{array}$ & $\begin{array}{c}60.06 \pm \\
1.04\end{array}$ & $\begin{array}{c}60.51 \pm \\
0.99\end{array}$ & $\begin{array}{c}62.37 \pm \\
1.50\end{array}$ & $\begin{array}{c}62.07 \pm \\
1.66\end{array}$ & $\begin{array}{c}60.98 \pm \\
1.71\end{array}$ & $\begin{array}{c}55.58 \pm \\
1.54\end{array}$ \\
\hline KEAPI & 96 & $\begin{array}{c}51.35 \pm \\
1.12\end{array}$ & $\begin{array}{c}53.98 \pm \\
1.60\end{array}$ & $\begin{array}{c}51.37 \pm \\
1.17\end{array}$ & $\begin{array}{c}50.87 \pm \\
1.21\end{array}$ & $\begin{array}{c}61.07 \pm \\
1.60\end{array}$ & $\begin{array}{c}60.33 \pm \\
1.85\end{array}$ & $\begin{array}{c}60.05 \pm \\
1.58\end{array}$ & $\begin{array}{c}56.85 \pm \\
1.48\end{array}$ \\
\hline TP53 & 200 & $\begin{array}{c}55.28 \pm \\
0.74\end{array}$ & $\begin{array}{c}54.38 \pm \\
0.75\end{array}$ & $\begin{array}{c}55.18 \pm \\
0.78\end{array}$ & $\begin{array}{c}55.02 \pm \\
0.79\end{array}$ & $\begin{array}{c}58.59 \pm \\
1.30\end{array}$ & $\begin{array}{c}57.87 \pm \\
1.26\end{array}$ & $\begin{array}{c}57.78 \pm \\
1.52\end{array}$ & $\begin{array}{c}57.14 \pm \\
1.48\end{array}$ \\
\hline$B R A F$ & 30 & $\begin{array}{c}55.38 \pm \\
1.74\end{array}$ & $\begin{array}{c}55.14 \pm \\
2.49\end{array}$ & $\begin{array}{c}56.59 \pm \\
1.76\end{array}$ & $\begin{array}{c}56.87 \pm \\
1.74\end{array}$ & $\begin{array}{c}55.26 \pm \\
2.66\end{array}$ & $\begin{array}{c}57.10 \pm \\
1.95\end{array}$ & $\begin{array}{c}56.23 \pm \\
2.14\end{array}$ & $\begin{array}{c}51.35 \pm \\
2.88\end{array}$ \\
\hline$P D G F R B$ & 15 & $\begin{array}{c}67.85 \pm \\
2.51\end{array}$ & $\begin{array}{c}69.75 \pm \\
2.79\end{array}$ & $\begin{array}{c}69.91 \pm \\
3.23\end{array}$ & $\begin{array}{c}70.06 \pm \\
2.97\end{array}$ & $\begin{array}{c}72.03 \pm \\
2.04\end{array}$ & $\begin{array}{c}72.78 \pm \\
2.48\end{array}$ & $\begin{array}{c}73.50 \pm \\
2.02\end{array}$ & $\begin{array}{c}52.13 \pm \\
3.83\end{array}$ \\
\hline ROS1 & 18 & $\begin{array}{c}58.29 \pm \\
3.58\end{array}$ & $\begin{array}{c}60.74 \pm \\
2.76\end{array}$ & $\begin{array}{c}51.27 \pm \\
3.20\end{array}$ & $\begin{array}{c}49.97 \pm \\
3.20\end{array}$ & $\begin{array}{c}59.44 \pm \\
4.69\end{array}$ & $\begin{array}{c}60.07 \pm \\
2.55\end{array}$ & $\begin{array}{c}61.45 \pm \\
4.54\end{array}$ & $\begin{array}{c}59.58 \pm \\
4.08\end{array}$ \\
\hline STK11 & 44 & $\begin{array}{c}53.98 \pm \\
1.76\end{array}$ & $\begin{array}{c}53.91 \pm \\
1.67\end{array}$ & $\begin{array}{c}53.56 \pm \\
1.83\end{array}$ & $\begin{array}{c}61.39 \pm \\
2.22\end{array}$ & $\begin{array}{c}58.41 \pm \\
1.51\end{array}$ & $\begin{array}{c}54.89 \pm \\
2.64\end{array}$ & $\begin{array}{c}59.22 \pm \\
2.30\end{array}$ & $\begin{array}{c}62.89 \pm \\
2.51\end{array}$ \\
\hline$M E T$ & 19 & $\begin{array}{c}61.22 \pm \\
2.31\end{array}$ & $\begin{array}{c}69.20 \pm \\
2.31\end{array}$ & $\begin{array}{c}63.14 \pm \\
2.39\end{array}$ & $\begin{array}{c}64.19 \pm \\
2.35\end{array}$ & $\begin{array}{c}66.58 \pm \\
3.70\end{array}$ & $\begin{array}{c}68.10 \pm \\
2.45\end{array}$ & $\begin{array}{c}63.68 \pm \\
2.76\end{array}$ & $\begin{array}{c}63.36 \pm \\
4.28\end{array}$ \\
\hline$A L K$ & 28 & $\begin{array}{c}53.55 \pm \\
2.23\end{array}$ & $\begin{array}{c}48.42 \pm \\
2.49\end{array}$ & $\begin{array}{c}62.64 \pm \\
3.05\end{array}$ & $\begin{array}{c}59.51 \pm \\
2.23\end{array}$ & $\begin{array}{c}50.32 \pm \\
2.88\end{array}$ & $\begin{array}{c}61.53 \pm \\
1.70\end{array}$ & $\begin{array}{c}58.71 \pm \\
1.95\end{array}$ & $\begin{array}{c}49.41 \pm \\
2.89\end{array}$ \\
\hline$D D R 2$ & 18 & $\begin{array}{c}57.17 \pm \\
3.81\end{array}$ & $\begin{array}{c}56.89 \pm \\
4.21\end{array}$ & $\begin{array}{c}57.38 \pm \\
3.80\end{array}$ & $\begin{array}{c}56.69 \pm \\
3.82\end{array}$ & $\begin{array}{c}55.63 \pm \\
4.30\end{array}$ & $\begin{array}{c}54.65 \pm \\
3.96\end{array}$ & $\begin{array}{c}52.56 \pm \\
4.37\end{array}$ & $\begin{array}{c}51.78 \pm \\
5.13\end{array}$ \\
\hline PIK3CA & 23 & $\begin{array}{c}49.14 \pm \\
1.78\end{array}$ & $\begin{array}{c}51.30 \pm \\
2.67\end{array}$ & $\begin{array}{c}51.07 \pm \\
2.11\end{array}$ & $\begin{array}{c}58.35 \pm \\
2.78\end{array}$ & $\begin{array}{c}53.90 \pm \\
3.27\end{array}$ & $\begin{array}{c}56.96 \pm \\
3.46\end{array}$ & $\begin{array}{c}58.01 \pm \\
3.18\end{array}$ & $\begin{array}{c}56.15 \pm \\
3.22\end{array}$ \\
\hline$E G F R$ & 49 & $\begin{array}{c}65.09 \pm \\
1.50\end{array}$ & $\begin{array}{c}61.48 \pm \\
2.49\end{array}$ & $\begin{array}{c}63.32 \pm \\
1.25\end{array}$ & $\begin{array}{c}64.90 \pm \\
1.97\end{array}$ & $\begin{array}{c}59.01 \pm \\
1.60\end{array}$ & $\begin{array}{c}59.69 \pm \\
1.56\end{array}$ & $\begin{array}{c}57.93 \pm \\
1.60\end{array}$ & $\begin{array}{c}49.05 \pm \\
1.97\end{array}$ \\
\hline
\end{tabular}

